\title{
Cryo-electron tomography study of detonation nanodiamonds hydrosols
}

\author{
A.A. Mikhutkin ${ }^{1}$, R.A. Kamyshinsky ${ }^{1,2}$, N.M. Kuznetsov ${ }^{1}$, S.N. Chvalun ${ }^{1}$, A.L. Vasiliev ${ }^{1,2}$

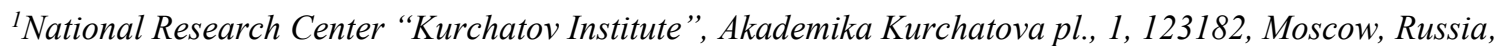 \\ ${ }^{2}$ Shubnikov Institute of Crystallography of FSRC “Crystallography and Photonics” RAS, Leninsky pr., 59, 119333 Moscow, Russia
}

\author{
Alex.Mikhutkin@gmail.com
}

In recent years serious attention has been focused on various applications of nanodiamonds obtained by detonation synthesis of carbon-containing explosives (detonation nanodiamonds - DNDs) [1]. DNDs find applications in biomedicine, development of novel liquid heat carriers and magnetic liquids. Thus, study of their rheological properties in various liquid media is of great importance [1]. Two rheological features of hydrosols have been recently discovered: the sharp increase in viscosity and the phase sol-gel transition at a relatively low filler concentration [2]. Such behaviour has been explained in the frame of percolation model based on network formation from the chains of faceted DND particles due to electrostatic interaction of facets [3]. The comprehension of DNDs structural organisation in various media is crucial for practical applications and it would be important to obtain a direct experimental evidence of DND chains existence in sol and network formation at the sol-gel transition. Such direct observation has been successfully performed by Cryo-Electron Tomography (Cyo-ET): the processes of bonding and agglomeration of the DND particles were observed, and the 3D spatial distribution and quantitative analysis, including fractal analysis, were accomplished on the basis of CyoET data. The obtained results explain the rheological properties of the DNDs hydrosols.

The study was focused on two types of DNDs hydrosols with positive and negative electrokinetic potential ( $\zeta$-potential) in the concentration range from 1.0 to $7.0 \mathrm{wt} \%$. Cryo-ET study was performed on Titan Krios 60-300 TEM/STEM (FEI, USA) at acceleration voltage of $300 \mathrm{kV}$.

The 3D models of positive and negative $\zeta$-potential DNDs were obtained and the 3D spatial distribution of DNDs was revealed [4]. The data demonstrate the formation of extended fractal structures and chains of individual faceted DND particles. The skeletonization procedure was applied in order to evaluate the bonding of objects and percolation. It was observed that DNDs with positive $\zeta$-potential form a percolation network. However, such network was not observed in DNDs hydrosols with negative $\zeta$-potential. This explains the differences in the rheological behavior at low concentrations of samples with different sign of $\zeta$-potential. The 2D and 3D fractal dimensions were calculated for the mass-fractal and fractal surface from the Cryo-ET data. The fractal dimensions are in good correlation with the small angle X-ray scattering (SAXS) data for the fractal dimension of a DNDs cluster. Moreover, the distances between DND particles were estimated after the 3D reconstruction of the specimen volume. Interparticle distance distributions showed, that the secondary maximum position qualitatively matches the interplanar distance between fractals at $1 \mathrm{wt} \%$ contain of DND particles in hydrosol measured by SAXS.

The results of 3D reconstruction and analysis based on Cryo-ET data allowed to explain observed features of the rheological behavior associated with DNDs agglomeration and chain formation.

[1] Shvidchenko, A.V., Eidelman, E.D., Vul', A.Ya. et al. (2019). Adv. Colloid Interface Sci. $268,64$.

[2] Vul', A.Ya., Eidelman, E.D., Aleksenskiy, A.E. et al. (2017). Carbon. 114, 242.

[3] Kuznetsov, N.M., Belousov, S.I., Stolyarova, D.Yu. et al. (2018). Diam. Relat. Mater. 83, 141.

[4] Kuznetsov, N.M., Belousov, S.I., Bakirov, A.V. et al. (2020). Carbon. 161, 486.

Keywords: 3D reconstruction and analysis; cryo-electron tomography; nanodiamonds hydrosols; fractal analysis; rheological properties

The detonation nanodiamonds hydrosols were kindly provided by Prof. Vul' A.Ya. and coworkers from Laboratory Physics for Cluster Structures of Ioffe Institute. This work was partially supported by Russian Foundation for Basic Researches, project 18-29-19117 mk. 